\section{ADDITIONS TO THE FAUNA OF PARASITIC WASPS (HYMENOPTERA: CHALCIDOIDEA) AND COCCOIDS (HEMIPTERA: COCCOIDEA) FROM THE ANDAMAN AND NICOBAR ISLANDS, INDIA, WITH ILLUSTRATIONS AND DIAGNOSIS}

\author{
Ankita Gupta ${ }^{1}$ \& Sunil Joshi ${ }^{2}$ \\ ${ }^{1,2}$ National Bureau of Agriculturally Important Insects (ICAR), P.B. No. 2491, H.A. Farm Post, Bellary Road, Hebbal, \\ Bengaluru, Karnataka 560024, India \\ ${ }^{1}$ drankitagupta7@gmail.com (corresponding author), 2 sunjoshi.pdbc@gmail.com
}

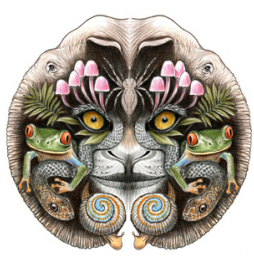

ISSN

Online 0974-7907

Print 0974-7893

OPEN ACCESS
Abstract: An illustrated account with diagnostic details of the reared and collected species of insect parasitoids along with scale insects and mealybugs from the Andaman and Nicobar Islands is presented. Twenty eight species of insect pests and parasitoids under two major orders Hemiptera and Hymenoptera are documented, of which 16 species are reported for the first time from these islands specifically from South and Middle Andamans. The parasitic wasps reared and collected belong to six families-Encyrtidae, Eulophidae, Chalcididae, Eucharitidae, Aphelinidae, and Pteromalidae, including 16 species under 12 genera. The majority of the species are primary or secondary parasitoids attacking many insect groups. The scale insects and mealybugs documented belong to four familiesCoccidae, Pseudococcidae, Diaspididae, and Cerococcidae, including 12 species under 11 genera. Information on the species distribution, host association, and brief taxonomical description of each species along with illustrations is provided for easy identification. Illustrations include habitus photographs of 16 species of parasitic wasps and 12 species of scales and mealybugs.

Keywords: Andamans, host record, mealybugs, parasitoids, scale insects.
The Andaman and Nicobar Islands consist of 349 islands situated in the Bay of Bengal in the northern Indian Ocean between $60-140^{\circ} \mathrm{N}$ and $920-940^{\circ} \mathrm{E}$. The insect fauna of the Andamans and Nicobar Islands is relatively less known in comparison with the explored and documented diverse fauna from the Indian mainland.

A compilation of abstracts covering 320 research publications dealing with agricultural research during the period 1978-1990 from the bay islands and a list of natural enemies of insect pests was documented (Bhumannavar et al. 1991a,b). A total of 41 species of parasitic wasps from the superfamily Chacidoidea of the order Hymenoptera were listed from the Andaman and Nicobar Islands (Noyes 2012). Insect herbivores and the natural enemies associated with mangroves of the Andaman and Nicobar Islands were also documented (Veenakumari et al. 1997).

Parasitic wasps play a key role in targeting insect pests of economic importance. To document parasitoids associated with scales and mealybugs, recent surveys

DOI: http://dx.doi.org/10.11609/JoTT.03360.4542-55 | ZooBank: urn:Isid:zoobank.org:pub:3CCF670A-EOCD-4170-BC81-B67AF91A45DF

Editor: T.C. Narendran, Prof. T.C. Narendran Trust for Animal Taxonomy, Kozhikode, India.

Date of publication: 26 July 2013 (online \& print)

Manuscript details: Ms \# 03360 | Received 22 September 2012 | Final received 28 June 2013 | Finally accepted 03 July 2013

Citation: Gupta, A. \& S. Joshi (2013). Additions to the fauna of parasitic wasps (Hymenoptera: Chalcidoidea) and coccoids (Hemiptera: Coccoidea) from the Andaman and Nicobar Islands, India, with illustrations and diagnosis. Journal of Threatened Taxa 5(11): 4542-4555; http://dx.doi.org/10.11609/JoTT.o3360.4542-55

Copyright: ( G Gupta \& Joshi 2013. Creative Commons Attribution 3.0 Unported License. JoTT allows unrestricted use of this article in any medium, reproduction and distribution by providing adequate credit to the authors and the source of publication.

Funding: The survey and research was sponsored by the Indian Council of Agricultural Research (NBAll Institute funds)

Competing Interest: None.

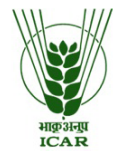

Acknowledgements: We gratefully acknowledge Dr. B.S. Bhumannavar, Director, National Bureau of Agriculturally Important Insects (ICAR), Bengaluru, India for encouraging us for research work. We are thankful to the Indian Council of Agricultural Research for providing financial support for the survey and research work. The first author is extremely thankful to Dr. P. Vishwakannan, D.F.O., Middle Andamans for the permission granted to survey and also for the kindness and generosity offered during the stay at Andamans. We also thank the Director, Central Agricultural Research Institute, Head, Division of Plant Protection and Dr. Ajanta Birah, former Sr. Scientist, CARI for facilitating our stay at CARI, Port Blair. 
were undertaken in two batches from the National Bureau of Agriculturally Important Insects, Bengaluru, India, to the Andaman and Nicobar Islands during the months of February and March, 2012. The islands surveyed were South and Middle Andamans covering Port Blair, Sipighat, Blooms Dale, Wandoor, Havelock Island, Neil Island, Lakshmanpur, Rangat, Jirkatang, Baratong, Mount Harriet, Kadamtala and Yerata.

We documented 28 species of insects and parasitoids under the two major orders Hemiptera and Hymenoptera. Out of these, 16 species of parasitic wasps belong to the order Hymenoptera under the super family Chalcidoidea, of which many are associated with mealybugs and scale insects and amongst them nine species of wasps are reported for the first time from these islands and two species are documented with new host records. Of the total 12 species of scales and mealybugs from the order Hemiptera, seven species are reported for the first time. The insects collected were reared to adult stage on the associated hosts. The parasitoids that emerged during the rearings were collected and documented.

\section{Material and Methods}

Sampling: This work is based on studies of nearly 160 specimens collected from South and Middle Andamans. The wasp specimens were collected using sweep nets, yellow pan traps, malaise traps, and the collection of parasitized hosts was followed by subsequent laboratory rearing. Malaise traps and yellow pan traps were set in 20 different locations. Parasitized hosts were collected and kept in the laboratory for parasitoid emergence. Wasp specimens were processed using Hexamethyldisilazane (Brown 1993) and card mounted and identified consulting (Hayat 1986, 1998, 2006; Mani 1989; Narendran 1989; Noyes \& Hayat 1994; Narendran \& Mini 2000; Xiao \& Huang 2001; Verma et al. 2002). The scale insects and mealybugs were collected from the infested host plants and preserved in $70 \%$ alcohol. Specimens were later mounted on slides following standard devised techniques (Sandlant 1978). Scale insects were identified following identification manual of family Coccidae (Hodgson 1994) and mealybugs were identified based on the keys for South Asia (Williams 2004). The wasp and coccid images were taken using Leica M 205 A stereozoom microscope with Leica DC 420 inbuilt camera using automontage software (version 3.8). All the specimens are deposited in the National Bureau of Agriculturally Important Insects, Bengaluru, India.

Sampling sites: South Andamans collection sites: Port Blair, CARI campus, Sipighat, Blooms Dale Farm, Neil
Island, Lakshmanpur, Mount Harriet, Havelock island, Kalapattar, and Jirkatong/Jirkatang. Middle Andamans include: Kadamtala and Rangat. For Baratong/Baratang: Middle Andamans is at its north and South Andamans at its south.

The GPS readings of all the surveyed sites are as follows: Sipighat $11.3656^{\circ} \mathrm{N}$ \& $92.40^{\circ} \mathrm{E}$; Blooms Dale Farm $11.798^{\circ} \mathrm{N} \& 92.26^{\circ} \mathrm{E}$; Neil Island $11.4994^{\circ} \mathrm{N}$ \& $93.93^{\circ} \mathrm{E}$; Lakshmanpur $11.3798^{\circ} \mathrm{N} \& 92.39^{\circ} \mathrm{E}$; Kadamtala $11.5029^{\circ} \mathrm{N}$ \& $92.39^{\circ} \mathrm{E}$; Mount Harriet $11.5029^{\circ} \mathrm{N}$ \& $92.25^{\circ} \mathrm{E}$; Havelock island, Kalapattar $13.0162^{\circ} \mathrm{N}$ \& $77.05^{\circ} \mathrm{E}$; Port Blair, CARI campus $11.3656^{\circ} \mathrm{N} \& 92.67^{\circ} \mathrm{E}$; Jirkatong/Jirkatang $11.3662^{\circ} \mathrm{N}$ \& $92.85^{\circ} \mathrm{E}$; Rangat $12.2113^{\circ} \mathrm{N} \& 92.41^{\circ} \mathrm{E}$; Baratong/Baratang $12.2113^{\circ} \mathrm{N} \&$ $92.46^{\circ} \mathrm{E}$; Yerata $12.2113^{\circ} \mathrm{N} \& 92.46^{\circ} \mathrm{E}$

\section{Order Hymenoptera \\ (Images 1-16) \\ Phylum: Arthropoda \\ Class: Insecta \\ Order: Hymenoptera \\ Super family: Chalcidoidea \\ Family Encyrtidae (Images 1-6)}

\section{Leptomastix nigrocincta Risbec (Image 1)}

Leptomastix nigrocincta Risbec, 1959: 27, F. Lectotype F (designated by Noyes \& Prinsloo, 1998): Madagascar (MNHN).

Material studied: NBAll/2012/Enc/Ana/dact/2, 10.iii.2012, one male and one female, Mount Harriet, sweep net, coll. Ankita Gupta.

Brief diagnosis: Female. Head yellowish, mandible orange with base yellowish; pedicel and flagellum brown; scape yellowish with dorsal margin brown; mesosoma orange; mid coxae brown, rest legs yellow; metasoma orange; F1 shorter than clava, $0.7 x$ clava; $F 6$ almost $2 x$ as long as wide; fore wing with linea clava interrupted not more than two lines of setae; marginal vein $0.8 x$ post marginal vein and $1.16 \mathrm{x}$ stigma vein.

Distribution: Throughout India including Andaman and Nicobar Islands (Hayat 2006).

Hosts: Coccidohystrix insolita (Sankaran) and Phenacoccus sp. (Noyes \& Hayat 1994).

Anagyrus diversicornis (Howard) Noyes, 2000: 34, 71 72, F, M, tax., des., distrib. (Image 2)

Copidosoma diversicornis Howard in Riley, Ashmead \& Howard, 1894: 92, F. Lectotype F (designated by Noyes, 1979: 147): West Indies, St Vincent (BMNH).

Brief diagnosis: Scape yellowish with a brown patch in middle; pedicel, F1, F3-F6 and first segment of clava dark 
brown; F2 and rest of clava white. Head and mesosoma dark brown; metasoma completely dark brown. Legs pale yellow with femora and tibia with brown infuscation dorsally; wings hyaline. Ovipositor exserted.

Material studied: NBAll/2012/Enc/Apo/bicol/1, 10.iii.2012, one female, Mount Harriet, sweep net, coll. Ankita Gupta.

Distribution: Andaman Islands (new record) and Tamil Nadu.

Hosts: Indet. coccids on Annona squamosa and pseudococcid on Solanum nigrum (Manickavasagam et al. 2001)

\section{Callipteroma sexguttata Motschulsky (Image 3)}

Callipteroma quinqueguttata Motschulsky, 1863: 36-37, F. Syntypes F: Sri Lanka, Mt. Patannas (ZMMU). Synonymy by Boucek, 1977:70.

Material studied: NBAll/2012/Enc/Call/sexg/1, 10.iii.2012, one female, Mount Harriet, sweep net, coll. Ankita Gupta.

Brief diagnosis: Female blackish-brown; forewings mostly dark brown with hyaline areas, normally hyaline at base, two hyaline spots below submarginal vein, one on anterior wing margin at apex of venation and one opposite on posterior wing margin, one distad of this in disc of wing and apex narrowly hyaline.

Distribution: India: Andaman Islands (new record), almost widely distributed throughout India (Hayat 2006).

Hosts: Birendracoccus saccharifolii on sugarcane from Pakistan (Noyes \& Hayat 2004).

\section{Lakshaphagus fusiscapus (Agarwal) (Image 4)}

Cheiloneurus fusiscapus Agarwal, 1965: 62, 63-65, F. Holotype F: India, Aligarh (ZDAMU).

Lakshaphagus fusiscapus (Agarwal): Hayat, 1981: 22 23, tax.

Material studied: NBAll/2012/Enc/Lak/fus/5, 10.iii. 2012, five females, ex. Cerococcus indicus (Maskell), Yerata, middle Andaman, coll. Ankita Gupta.

Brief diagnosis: Head and mesosoma dark orange; mid and hind tibia without brownish bands; funicle segments quadrate to slightly longer than broad, F1-F3 brownish, broader than long; F4-F6 whitish, clava dark brown; metasoma dark brown and metallic. Scutellum in apical third with scale like dark brown setae, the apical pair much longer.

Distribution: India: Andaman Islands (new record), Karnataka, Madhya Pradesh, Uttar Pradesh, Rajasthan, Maharashtra and Tamil Nadu (Hayat 2006).

Hosts: Cerococcus sp., Ceroplastodes sp. and
Planococcus citri. Full details given in Hayat 2006

Remarks: Lakshaphagus fusiscapus (Agarwal) is a new record from Hibiscus sp. scale - Cerococcus indicus (Maskell) from Yerata in Middle Andamans.

Family Eulophidae

Elasmus noyesi Verma \& Hayat (Image 5)

Elasmus noyesi Verma \& Hayat in Verma et al., 2002: 36, 249, 258-259, F: BMNH.

Material studied: NBAll/2012/Eul/Elas/nov/1, 10.iii.2012, one female, Rangat, Malaise trap, coll. Ankita Gupta.

Brief diagnosis: Body black with greenish-blue shine, intense on fronto vertex, pronotum, mesosoma, propodeum, dorsal sides of hind coxae. Scape pale brown with dark brown infuscations dorsally, flagellum dark brown. Scutellum with a yellowish longitudinal narrow band on each side. Coxae almost black. Wings hyaline. Hind tibia with diamond shaped patterns. Metasoma reddish-brown basally, reddish-brown colouration more conspicuous laterally; apex black. Fore femora yellowish except brown infuscation at base on dorsal margin. Mid and hind femora dark brown, yellowish at apex and base.

Distribution: Andaman Islands (new record), Karnataka and Kerala (Noyes 2012).

\section{Elasmus flavescens Verma \& Hayat (Image 6)}

Elasmus flavescens Verma \& Hayat in Verma et al., 2002: 36, 251, 284-285, F: BMNH.

Material studied: NBAll/2012/Eul/Elas/flav/1, 10.iii.2012, one female, Mount Harriet, sweep net, coll. Ankita Gupta.

Brief diagnosis: Body yellow with following brown to black parts: large portion of frontovertex surrounding ocelli extending in front of anterior ocellus; pronotum largely black (except sides and apex yellow); axillae and tegulae brown; metanotum dark brown; propodeum metallic blue. Metasoma yellow with basal half of T1 black with metallic green shine; tergite T3 with a brown spot medially; T6 largely blackish, T7 yellow; scape 3.5X as long as broad.

Distribution: Andaman Islands (new record), Karnataka and Kerala (Hayat 2006).

Hosts: Unknown.

Family Chalcididae (Images 7-11)

Antrocephalus mitys (Walker) Boucek, 1976: 345-346. (Image 7)

Halticella mitys Walker, 1846: 81, F, Mauritius (BMNH). 

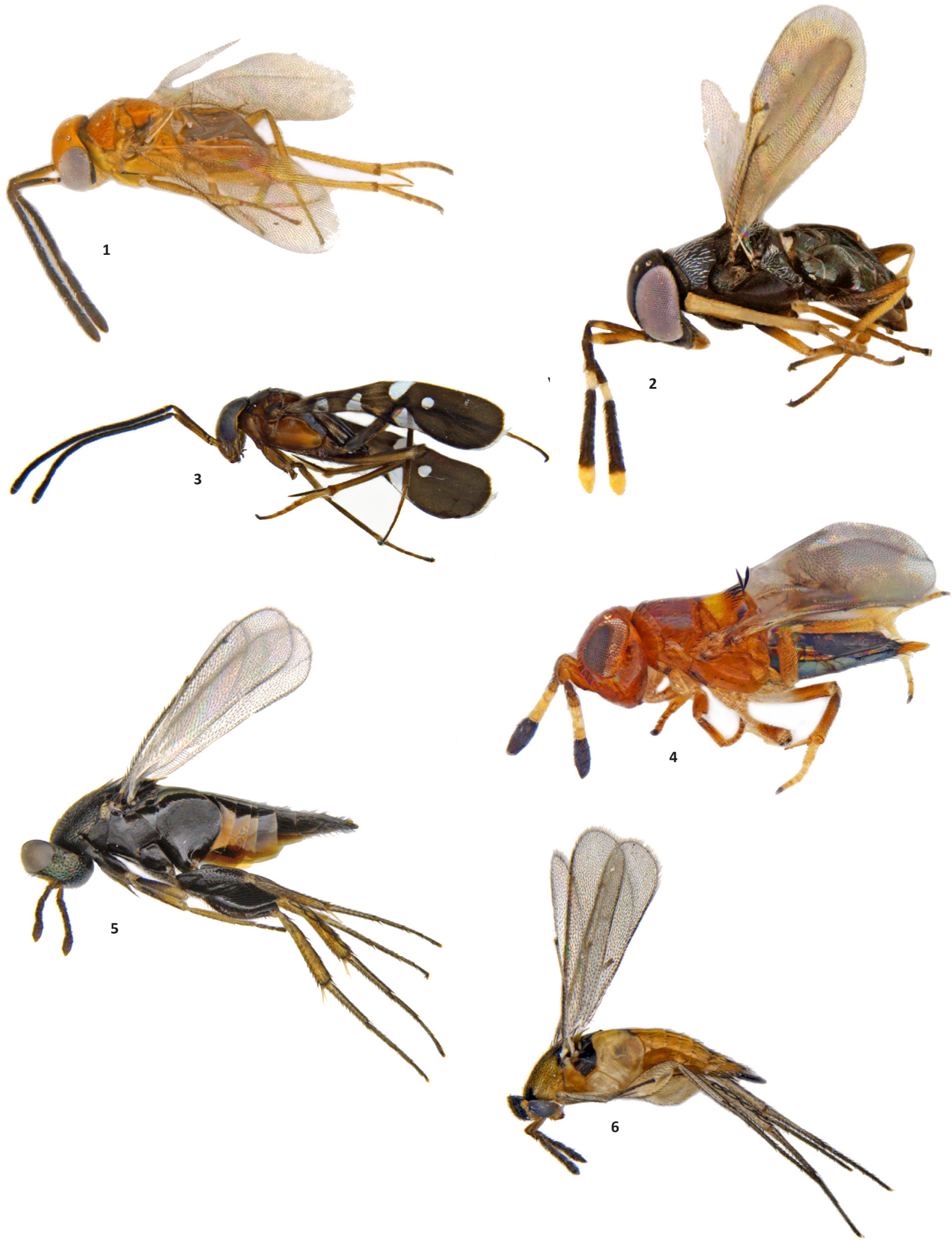

Images 1-6. 1 - Leptomastix nigrocincta Risbec; 2 - Anagyrus diversicornis (Howard); 3 - Callipteroma sexguttata Motschulsky;

4 - Lakshaphagus fusiscapus (Agarwal); 5 - Elasmus noyesi Verma \& Hayat; 6 - Elasmus flavescens Verma \& Hayat. (C) Ankita Gupta 
Material studied: NBAll/2012/Chal/Ant/mit/4, 05.iii.2012, four females, Sippighat, Malaise trap, coll. Ankita Gupta.

Brief diagnosis: Female black; legs rufous. Antennae largely black (except F1 and F2 dark brown). Hind femur reddish; metasoma longer than mesosoma; post marginal vein subequal to marginal. Propodeum with distinct lateral teeth. First tergite of metasoma smooth and shiny. Hind femur with inner basal tooth; scutellum with two teeth at apical margin and median fovea.

Distribution: India: Andaman Islands (new record), Kerala, Tripura and Uttar Pradesh (Noyes 2012)

Hosts: Mostly from the family Pyralidae- Chilo partellus Swinhoe, Corcyra cephalonica (Stainton), Lamida moncusalis Walker and Apomyelois (Ectomyelois) ceratoniae (Zeller) (Noyes 2012).

\section{Brachymeria ryukyuensis Habu (Image 8)}

Brachymeria ryukyuensis Habu, 1963: 115, Japan

Material studied: NBAll/2012/Chal/Bra/ryuk/3, 10.iii.2012, three females and males, Kadamtala, sweep net collection, coll. Ankita Gupta.

Brief diagnosis: Body black. Antennae black. Tegulae pale yellow; coxae and trochanters black; hind femora with apical yellow patch; fore and mid tibiae pale yellow with incomplete black patch in the middle; hind tibia black with sub basal and apical yellowish-white patch. All tarsi pale yellow. Pre orbital carinae not prominent and post orbital carinae present; apex of scutellum slightly pointed and round in shape.

Distribution: India: Andaman and Nicobar Islands, Tripura and Kerala (Noyes 2012).

Hosts: Unknown.

\section{Dirhinus anthracia Walker (Image 9)}

Dirhinus anthracia Walker, 1846: 85, BMNH, Philippines.

Material studied: NBAll/2012/Chal/Dir/anth/1, 10.iii.2012, one female, malaise trap, Kadamtala. coll. Ankita Gupta.

Brief diagnosis: Black. Antenna rufous. Fore and mid leg rufous, hind legs black. Straie on first metasomal tergite reaching $1 / 3^{\text {rd }}$ of length, remaining tergite smooth and shiny. Head and mesosoma prominently setose, setae pale white. Fore wings slightly fuscous. Propodeum rugose with submedian and sublateral carinae.

Distribution: India: Andaman Islands (new record), Madhya Pradesh, Punjab, Tripura, Uttar Pradesh and Manipur (Noyes 2012).

Hosts: Calliphora villosa, Dacus cucurbitae, Dacus ferrugineus, Musca domestica, Sarcophaga aurifrons, Placoptera reflexa, Brachartona catoxantha, Pyrausta machaeralis (Narendran 1989). Hapalia machaeralis, Chortoicetes terminifera and Bombyx mori (Noyes, 2012).

Hockeria polycarinata Narendran (Image 10)

Hockeria polycarinata Narendran, 1989: 89-90, F, USNM, Java.

Material studied: NBAll/2012/Chal/Hock/poly/1, 10.iii.2012, 1 female, Malaise trap, Rangat, coll. Ankita Gupta.

Brief diagnosis: Scape and first three funicular segments yellowish brown; tegulae yellowish-brown; Hind coxa black; hind femur balck with apex and base reddish-brown hind tibia reddish-brown with middle ventral margin black also extending to dorsal region. Hind trasi reddish-brown. First metasomal tergite with small basal carinae, inclined laterally on both sides; present in basal $1 / 3^{\text {rd }}$ of first tergite.

Distribution: Andaman Islands (new record) and Indonesia (Java) (Noyes, 2012).

Hosts: Unknown.

\section{Hockeria bifasciata Walker (Image 11)}

Hockeria bifasciata Walker, 1834: Lectotype F, designated by Boucek, 1992: 53, 61, BMNH, France

Material studied: NBAll/2012/Chal/Hock/bifa/2, 08.iii.2012, two males, Malaise trap, Sippighat, coll. Ankita Gupta.

Brief diagnosis: Body black, antennae dark brown. Legs including coxae black except for faint brown apices of tibiae. Head and mesosoma distinctly punctuate and setose. Scutellum entire. Propodeum with distinctly carinae, faint transverse carinae present inside the areola of propodeum. Metasoma sessile, smooth and shiny. Basal $1 / 3^{\text {rd }}$ of first tergum rugose with small carinae; lateral sides of second tergum with fine punctures. Marginal vein longest; post marginal vein rudimentary; stigma vein short and curved, $0.3 x$ marginal vein.

Distribution: India: Andaman Islands (new record) and Kerala (Noyes 2012).

Hosts: Unknown from India.

Family Eucharitidae (Images 12-16)

Schizaspidia andamanensis (Mani) (Image 12)

Kapaloides andamanensis Mani, 1942: 156, M, ZSIC, India-Andaman and Nicobar islands.

Schizaspidia andamanensis (Mani): Narendran, 1986: 53.

Material studied: NBAll/2012/Euch/Schi/and/3, 

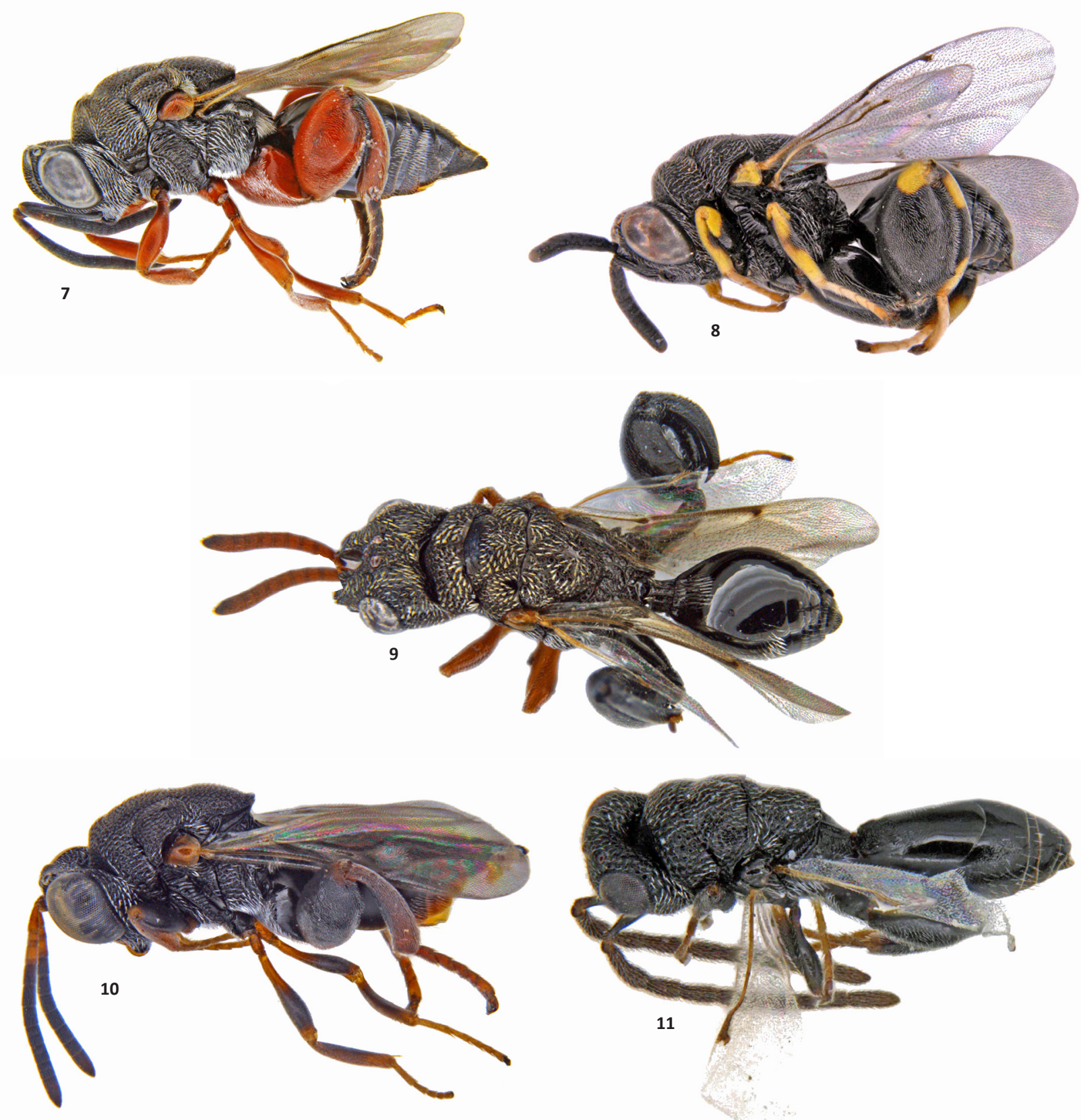

Images 7-11. 7 - Antrocephalus mitys (Walker); 8 - Brachymeria ryukyuensis Habu; 9 - Dirhinus anthracia Walker; 10 - Hockeria polycarinata Narendran; 11 - Hockeria bifasciata Walker. (c) Ankita Gupta

11.iii.2012, three males, Mount Harriet, yellow pan trap, coll. Ankita Gupta.

Brief diagnosis: Body dark metallic green; scape and pedicel yellowish-brown; coxae concolorous with mesosoma. Scutellum with a median furrow. Legs light brown. Scape $2.7 x$ as long as wide; head $1.4 x$ as wide as long. First flagellar segment with a branch; branches of flagellar segments only slightly flattened and cylindrical. Petiole longer than coxa in male. Scutellar processes
$1.17 x$ longer in male than in female; almost of same width throughout except at extreme apex. Tines of the scutellar fork slightly convergent; bending in curve towards each other; carinae clearer in male than in female.

Distribution: Andamans (Mount Harriet - new record) and Port Blair (Noyes 2012).

Hosts: Unknown. 
Family Aphelinidae (Images 12-16)

Marietta leopardina Motschulsky (Image 13)

Marietta leopardina Motschulsky, 1863: 52, F, M, ZMMU, Sri Lanka.

Material studied: NBAll/2012/Aph/Mar/leop/14, 06.iii.2012, two females, ex. indet. scales on ornamental plant from Rangat, middle Andaman, coll. Ankita Gupta.

Brief diagnosis: Body 0.8-0.9 mm, pale coloured, with a beautiful pattern of dark bands or spots on head, mesosoma, metasoma and legs. Antenna six-segmented in female and five-segmented in male. Fore wing conspicuously maculated with a dark and light pattern of setae; marginal vein about $1.25 x$ as long as submarginal vein, postmarginal absent, stigmal vein punctiform.

Distribution: Throughout India including Andaman and Nicobar Islands and the Oriental region (Noyes 2012).

Hosts: Commonly recorded as a hyperparasitoid associated with mealybugs and scales. Complete host details mentioned in Hayat 1998.

Coccophagus ceroplastae (Howard, 1895) (Image 14)

Aneristus ceroplastae Howard, 1895: 351, F, ?USNM, Jamaica.

Coccophagus ceroplastae (Howard): Hayat, 1998: 162.

Material studied: NBAll/2012/Aph/Cocc/cero/11, 06.iii.2012, eight females mounted on card and several preserved in $70 \%$ alcohol, ex. Parasaissetia nigra (Nietner) on Hibiscus sp. from Rangat, middle Andaman, coll. Ankita Gupta.

Brief diagnosis: Body dark brown to black, mesosoma including scutellum, dark with a distinct metallic violet tinge, metasoma dark; antenna brown to dark brown. Flagellum somewhat flattened. Fore wing hyaline, strongly infuscate behind marginal and stigmal veins, infuscation distally convex; basal cell with few setae; hind wing hyaline.

Distribution: India: Well distributed throughout India.

Host: Reported from scales (Hemiptera: Coccoidea) viz., Coccus viridis, $C$. hesperidum, Saissetia coffeae, Ceroplastes spp., Pulvinaria psidii, P. polygonata (Coccidae), etc. Scales and mealybugs from the families Diaspididae and Pseudococcidae have also been recorded as hosts (Hayat, 1998).

Remarks: In our study the parasitoid Coccophagus ceroplastae (Howard) and Coccophagus sp. was bred from Coccus hesperidium L. scale from Jirkatang, Middle Andamans and from Parasaissetia nigra (Nietner) from Rangat.
Coccophagus longipedicellus Shafee (Image 15)

Coccophagus longipedicellus Shafee, 1972: 25, F, ZAMU, India - Tumkur.

Material studied: NBAll/2012/Aph/Cocc/long/2, 06.iii.2012, two females, ex. Parasaissetia nigra (Nietner) on Hibiscus sp. from Rangat, Middle Andaman, coll. Ankita Gupta.

Brief diagnosis: Body orange yellow with metasoma having a dark brown cross band in about middle of dorsum, wings hyaline. First funicle segment short, about $1.5 x$ as broad as long.

Distribution: India: Andaman and Nicobar Islands and Karnataka (Noyes 2012).

Hosts: Parasaissetia nigra (Nietner) on Hibiscus sp. is new host record from Andamans. Earlier recorded from Coccus sp.on Mangifera indica L.

Family Pteromalidae (Images 12-16)

\section{Cephaleta australiensis (Howard) (Image 16)}

Anysis australiensis Howard, 1896: 167, USNM.

Cephaleta australiensis (Howard): Boucek et al. 1979: 438.

Material studied: NBAll/2012/Ptr/Ceph/aust/2, four females and three males, 06.iii.2012, ex. Cerococcus indicus (Cerococcidae) on Hibiscus sp. from Yerata, Middle Andaman, coll. Ankita Gupta.

Brief diagnosis: Body with shiny metallic shine, antenna dark brown except scape (yellowish-brown), legs yellowish-brown except all coxae black, tarsi dark brown, first pair of legs slightly darker brown. Head much wider than mesosoma. Male antenna nine-segmented with all funicular segments longer than broad, first one curved and longer than scape. Scutellum with silvery white hairs in apical third.

Distribution: Widely distributed throughout India (Andaman and Nicobar Islands, Andhra Pradesh, Bihar, Delhi, Kerala, Tamil Nadu, Karnataka, Orissa, Assam, Maharashtra, Uttar Pradesh, West Bengal). Oriental region. Nearctic (Noyes 2012).

Host: Commonly collected in association with several species of Coccoidea (Sternorrhyncha) including Coccus spp., Ceroplastes spp., Saissetia spp., Drepanococcus chiton, Pulvinaria psidii (Coccidae); Cerococcus indicus (Cerococcidae); Ferrisia virgata (Pseudococcidae) and Asterolecaniidae (Noyes 2012).

Remarks: So far only two species of the family Pteromalidae are reported from the Andaman and Nicobar Islands - $C$. australiensis and $C$. brunniventris Motschulsky, the latter one is mentioned as recorded from Andamans in the recent checklist by Sureshan, 


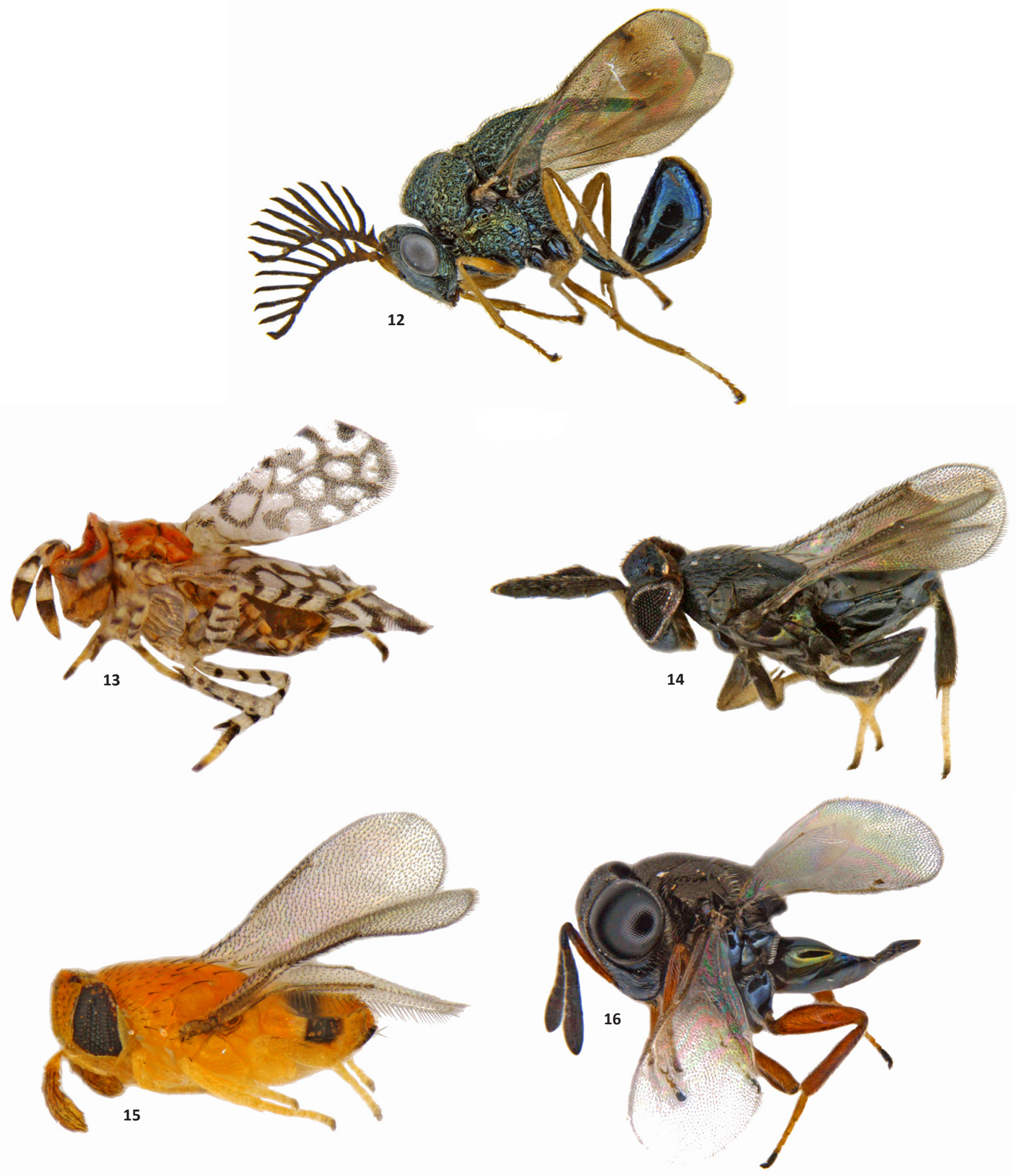

Images 12-16. 12 - Schizaspidia andamanensis (Mani); 13 - Marietta leopardina Motschulsky; 14 - Coccophagus ceroplastae (Howard); 15 - Coccophagus longipedicellus Shafee; 16 - Cephaleta australiensis (Howard). @ Ankita Gupta 
2012.

\section{Order Hemiptera}

(Images 17-28)

Phylum: Arthropoda

Class: Insecta

Order: Hemiptera

Super family: Coccoidea

Family Coccidae

\section{Coccus hesperidum Linnaeus (Image 17)}

Coccus hesperidum Linnaeus, 1758: 455. F, Europe: Syntypes, F.

Material studied: NBAll/2012/Coc/Cocc/hesp/12, 21.ii.2012, five females, Havelock Island, indet. climber (Cucurbitaceae), coll. Sunil Joshi.

Brief diagnosis: Body broadly oval to round; flat to slightly convex in lateral view; body yellowish-green to yellowish-brown, usually with small brown flecks scattered on dorsum. Mounted specimen with enlarged dorsal setae having acute or slightly rounded apices; ventral tubular ducts between middle legs, with a few (2-3) near base of hind legs; dorsal submarginal tubular ducts present around body margin.

Distribution: Andaman (new record), Bihar, Goa, Gujarat, Jammu and Kashmir, Kerala, Karnataka, Tamil Nadu, Tripura, Uttar Pradesh and West Bengal (Varshney, 1992).

\section{Parasaissetia nigra (Nietner) (Image 18)}

Lecanium nigrum Nietner, 1861: 9. F, Sri Lanka [=Ceylon]: on coffee. Syntypes, F.

Parasaissetia nigra Takahashi, 1955d: 26.

Material studied: NBAll/2012/Coc/Par/nig/13, 06.iii.2012, two females, Rangat, middle Andaman, coll. Ankita Gupta.

Brief diagnosis: Mature females with reticulate pattern on dorsum; marginal setae enlarged, fimbriate; without subdiscal or discal setae on anal plates; dorsal setae often capitate.

Distribution: Andaman (recorded earlier), Bihar, Tamil Nadu and West Bengal (Varshney 1992).

\section{Drepanococcus sp. (Image 19)}

Brief diagnosis: Marginal setae spinose; single long seta laterad of each spiracle; without dorsal setae; tubular ducts present over entire venter; without elongate prevulvar setae.

Material studies: NBAll/2012/Coc/Dre/sp/15, 21.ii.2012, eight females, Havelock Island, Annona sp., coll. Sunil Joshi
Distribution: Different species under this genus have been recorded throughout India (Ben-Dov et al. 2012).

Family Pseudococcidae

Antonina graminis (Maskell) (Image 20)

Sphaerococcus graminis Maskell, 1897: 244. F, Hong Kong: on grass. Lectotype $\mathrm{F}$, by subsequent designation Williams, 1985: 45. Type depository: Auckland: New Zealand Arthropod Collection, Landcare Research, New Zealand.

Antonina graminis Fernald, 1903b: 121.

Material studied: NBAll/2012/Pse/Ant/gram/16, 21.ii.2012, several females, Havelock island, indet. grass (Poaceae), coll. Sunil Joshi.

Brief diagnosis: Legs absent or represented by sclerotized pockets; antennae reduced to 2 or 3 segments; spiracles with trilocular pores present in sclerotized band surrounding spiracular atrium. Clusters of discoidal pores in ventrosubmarginal areas of abdominal segments II or III to VII or VIII; without dorsal and dorsomarginal band of multilocular pores; abdominal segments not forming sclerotized plate-like structures on segments III or IV to VIII; anal ring at apex of internal tube.

Distribution:Andaman (new record), Bihar, Karnataka, Maharashtra, Odisha, Tamil Nadu, Uttar Pradesh and West Bengal (Varshney 1992).

\section{Saccharicoccus sacchari (Cockerell) (Image 21)}

Dactylopius sacchari Cockerell, 1895: 195. F, Trinidad: St Ann's, on sugar-cane. Syntypes, F. Type depository: Washington: United States National Entomological Collection, U.S. National Museum of Natural History, District of Columbia, USA.

Saccharicoccus sacchari Ferris, 1950: 217. Change of combination.

Material studied: NBAll/2012/Pse/Sac/sac/14, 23.ii.2012, two females, Neil island, Saccharum officinarum L., coll. Sunil Joshi.

Brief diagnosis: Circulus hour-glass shaped; small discoidal pores surrounding hind coxae; cerarii restricted to anal lobes; multilocular pores present on dorsum and venter. Antennae usually seven segmented. Anterior and posterior pairs of ostiole present. Anal ring located at the apex of abdomen bearing six setae. Multilocular disc pores present mainly on the anterior and posterior margins of all the abdominal segments. Oral collar tubular ducts minute and are present in transverse rows on the abdomen.

Distribution: Andaman (new record), Arunachal Pradesh, Bihar, Gujarat, Karnataka, Madhya Pradesh, Maharashtra, Orissa, Punjab, Tamil Nadu, Uttar Pradesh, 

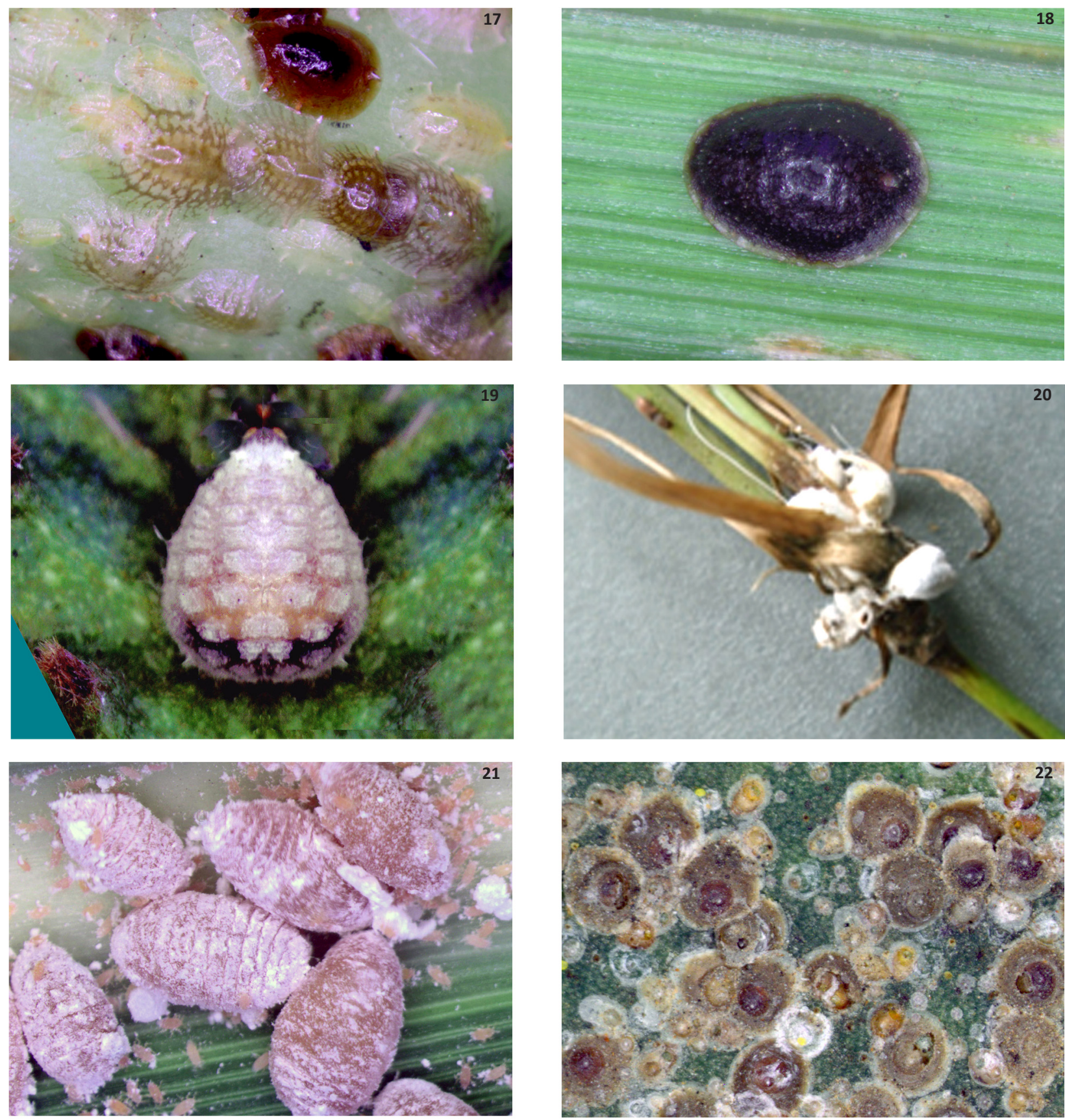

Images 17-22. 17 - Coccus hesperidum Linnaeus; 18 - Parasaissetia nigra (Nietner); 19 - Drepanococcus sp., Fig.20. Antonina graminis (Maskell); 21 - Saccharicoccus sacchari (Cockerell); 22 - Hemiberlesia lataniae (Signoret). @ Sunil Joshi

West Bengal (Varshney 1992).

\section{Family Diaspididae}

Hemiberlesia lataniae (Signoret) (Image 22)

Aspidiotus lataniae Signoret, 1869: 124. France, Syntypes, F. Type depository: Vienna: Naturhistorisches Museum Wien, Austria. Described: female and first instar.
Hemiberlesia crawii; Cockerell, 1899: 396.

Hemiberlesia lataniae; Borchsenius, 1966: 306.

Material studied: NBAll/2012/Dia/Hem/lat/17, 23.ii.2012, 12 females, Neil island, Pisidium guajava L., coll. Sunil Joshi.

Brief diagnosis: slide-mounted adult female membranous, and broadly pyriform. Pygidium with large median lobes close together, almost touching; 
second and third lobes reduced, unsclerotized, anal opening very large and its diameter about equal to length of median lobes, situated near posterior margin, of pygidium; paraphyses shorter than the lobes, present only on the margin between the third lobes, Perivulvar pores present.

Distribution: Andaman (Recorded earlier), Assam, Karnataka, Maharashtra, Odisha, Tamil Nadu and West Bengal (Varshney 1992).

\section{Abgrallaspis cyanophylli (Signoret) (Image 23)}

Aspidiotus cyanophylli Signoret, 1869: 119. F, France: Paris, Luxembourg Garden, on Cyanophyllum magnificum, Syntypes, F. Type depository: Vienna: Naturhistorisches Museum Wien, Austria.

Abgrallaspis cyanophylli; Balachowsky, 1948: 308.

Brief diagnosis: Body of slide-mounted adult female membranous and pyriform, with a well-developed eye spine present on each side, pygidium with large median lobes, quite small but well-developed, rounded second lobes and small (often pointed) third lobes; anal opening-large, situated near the posterior margin of the pygidium; paraphyses shorter than the lobes.

Material studied: NBAll/2012/Dia/Abg/cya/17, 23.ii.2012, two females, Neil island, Pisidium guajava L., coll. Sunil Joshi.

Distribution: Andaman (new record), all other states of India (Varshney 1992).

\section{Aonidiella aurantii (Maskell) (Image 24)}

Aspidiotus aurantii Maskell, 1879: 199. F, New Zealand: Governors Bay, on oranges and lemons imported to New Zealand from Sydney. Syntypes, F. Type depository: Auckland: New Zealand Arthropod Collection, Landcare Research, New Zealand.

Aonidiella aurantii; Berlese, 1895a: 125.

Brief diagnosis: Prosoma becoming highly sclerotised and expanded with maturity; eventually the lateral lobes may project further posteriorly than the apex of the pygidium. Abdominal segment lacking any prepygidial macroducts. Pygidium with paraphyses present, most of them not longer than median lobes.

Material studied: NBAll/2012/Dia/Aon/aur/18, 21.ii.2012, eight females, Havelock island, Citrus sp., coll. Sunil Joshi.

Distribution: Andaman (new record), Bihar, Punjab, West Bengal (Varshney 1992).

\section{Pinnaspis aspidistrae (Signoret) (Image 25)}

Chionaspis aspidistrae Signoret, 1869: 443. F, France: Paris, on Aspidistra sp. Holotype female. Type depository:
Vienna: Naturhistorisches Museum Wien, Austria.

Pinnaspis aspidistrae; Lindinger, 1912: 79.

Brief diagnosis: Body of slide-mounted adult female narrow and elongate, membranous, without lateral tubercles. Pygidium with well-developed median lobes without setae or gland spines between them; second lobes usually smaller than median lobes. Abdominal segment 5 with 1-3 submarginal macroducts on each side. Median lobes protrude as long as second lobes.

Material studied: NBAll/2012/Dia/Pin/asp/20, 22.ii.2012, eight females, Andaman Island, Areca sp., coll. Sunil Joshi

Distribution: Andaman (new record), Karnataka, Kerala, Madhya Pradesh, Maharashtra, Tamil Nadu and Tripura (Varshney 1992).

\section{Pinnaspis strachani (Cooley) (Image 26)}

Hemichionaspis minor strachani Cooley, 1899: 5455. F, Nigeria: Abeokuta, Type depository: Washington: United States National Entomological Collection, U.S. National Museum of Natural History, District of Columbia, USA.

Pinnaspis minor strachani; Kuwana, 1926: 40.

Pinnaspis strachani; Ferris \& Rao, 1947: 39.

Material studied: NBAll/2012/Dia/Pin/str/22, 21.ii.2012, three females, Havelock Island, Citrus sp., coll. Sunil Joshi.

Brief diagnosis: Body of slide-mounted adult female narrow and elongate, membranous, without lateral tubercles, pygidium with well developed median lobes without spines between them, abdominal segment 5 with 1-3 marginal macroducts. Median lobe protrude beyong second lobes.

Distribution: Andaman (recorded earlier), Andhra Pradesh, Jammu and Kashmir, Karnataka, Kerala, Tamil Nadu, Tripura and West Bengal (Varshney 1992).

\section{Family Cerococcidae}

\section{Cerococcus indicus (Maskell) (Image 27)}

Eriococcus paradoxus indica Maskell, 1897: 318. F, India: Uttar Pradesh, Saharanpur, on Helicteres isora. Syntypes, F. Type depositories: Auckland: New Zealand Arthropod Collection, Landcare Research, New Zealand, and London: The Natural History Museum, England, UK.

Notes: Lambdin \& Kosztarab (1977) state that topotype material was examined.

Cerococcus indicus Green, 1910: 5.

Material examined: NBAll/2012/Cer/Cer/ind/19, 21.ii.2012, six females, Havelock island, Hibiscus sp., coll. Sunil Joshi.

Brief diagnosis: Cribriform plates and 8-shaped 

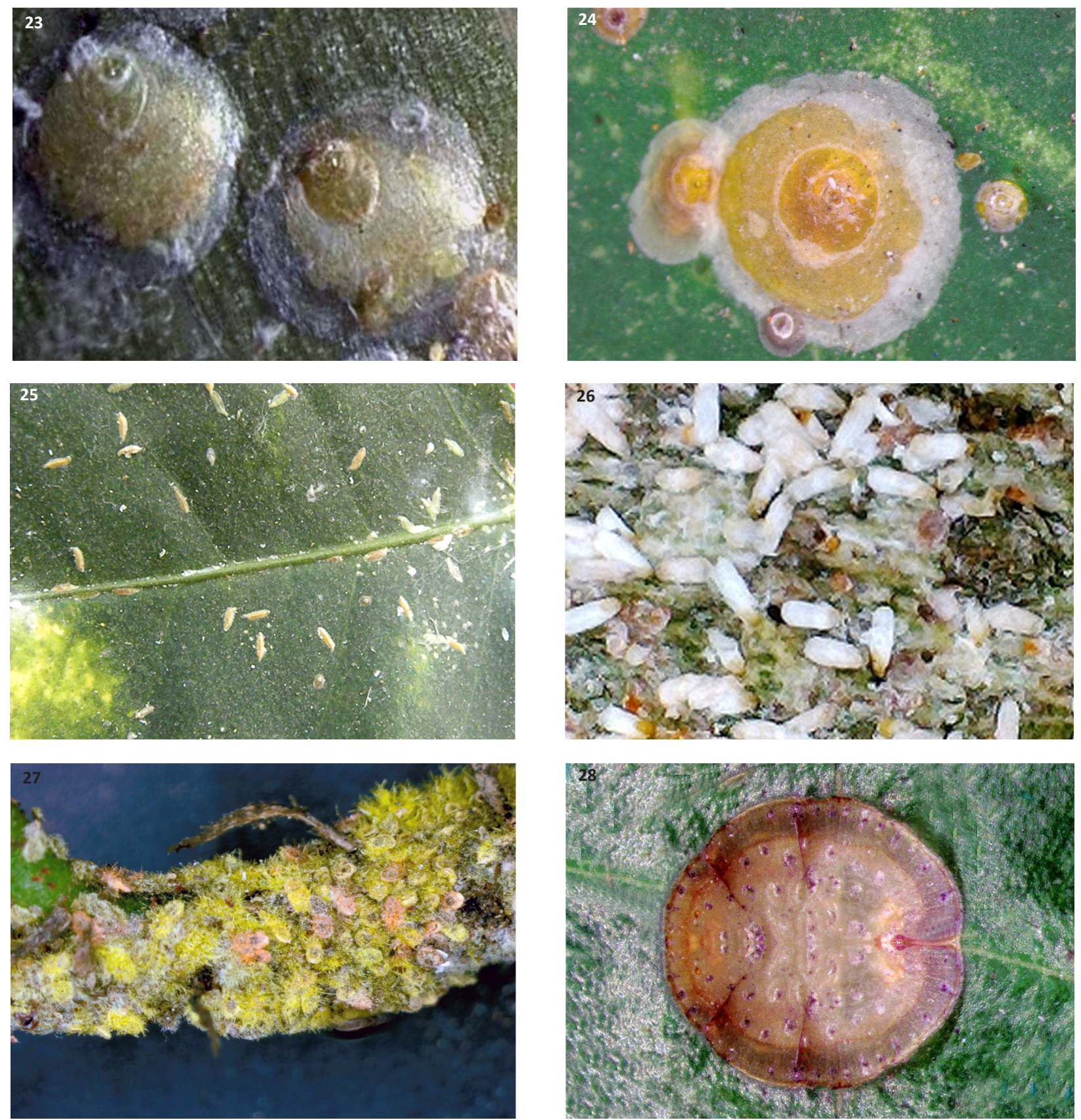

Images 23-28. 23 - Abgrallaspis cyanophylli (Signoret); 24 - Aonidiella aurantii (Maskell); 25 - Pinnaspis aspidistrae (Signoret); 26 - Pinnaspis strachani (Cooley); 27 - Cerococcus indicus (Maskell); 28 - Paralecanium sp. @ Sunil Joshi

pores present; spiracular furrows usually present, posterior furrow usually divided; single anal plate; antennae 1-segmented; legs absent or represented by unsegmented sclerotized spine; anal lobes usually protruding; labium 3-segmented.

Distribution: Andaman (recorded earlier), Andhra Pradesh, Bihar, Kerala, Karnataka, Madhya Pradesh, Maharashtra, Odisha, Tamil Nadu, Tripura, Uttar Pradesh and West Bengal (Varshney 1992).
Family Coccidae

Paralecanium sp. (Image 28)

Material examined: NBAll/2012/Coc/Par/sp/21, 22.ii.2012, three females, Andaman Island, coll. Sunil Joshi

Brief diagnosis: Adult female flat, broadly rounded, margin indented at stigmatic areas. Colour dark castaneous, with a narrow hyaline boarder and with hyaline canals extending inwards from each stigmatic 
cleft. Both antenna and legs wanting. Mounted female with fan shaped flabellae.

Distribution: Andaman (new record), the species of Paralecanium have been recorded from south-east Asia.

Remarks: The species that resembles to the species collected in present study is Paralecanium mancum Green. Diagnostic characters of this species are as follows. Colour dark castaneous, with a narrow hyaline border and with hyaline canals extending inwards from each stigmatic cleft. Dorsum with more or less well defined, thin, colourless, polygonal, waxy plates. Eye spots conscious; at some distance from the margin. Antennae relatively short, articulation obscure. Limbs entirely wanting. Anal operculum sub-quadrate, the base of each valve slightly shorter than its outer edge. Stigmatic spines three, blunt, the median spine usually shorter than the other two. Marginal flabellae subtriangular in form, the outer edge flatly curved, the converging slides approximately straight.

\section{REFERENCES}

Agarwal, M.M. (1965). Taxonomy of encyrtid parasites (Hymenoptera: Chalcidoidea) of Indian Coccoidea. Acta Hymenopterologica, Tokyo 2: 63.

Ben-Dov, Y., D.R. Miller \& G.A.P. Gibson (2012). ScaleNet, <http:// www.sel.barc.usda.gov/scalenet/lifehist.htm> (Accessed June $12^{\text {th }}$, 2012). Bhumannavar, B.S., P. Gangopadhyay, B. Gangwar \& A.K. Bandyopadhyay (1991a). Abstracts of agricultural research in Bay islands. Central Agricultural Research Institute. Port Blair, 76pp.

Bhumannavar, B.S., P. Mohanraj, H.R. Ranganath, T. Jacob \& K. Bandyopadhyay (1991b). Insects of Agricultural Importance in Andaman and Nicobar Islands. Research bulletin, Central Agricultural Research Institute, Port Blair, 6, 49pp.

Bouček, Z. (1976). Changes in the classification of some African Chalcidoidea (Hymenoptera). Journal of the Entomological Society of Southern Africa 39: 345-355.

Bouček, Z. (1977). A faunistic review of the Yugoslavian Chalcidoidea (Parasitic Hymenoptera). Acta Entomologica Jugoslavica 13: 1-145.

Bouček, Z. (1992). The New World genera of Chalcididae. Memoirs of the American Entomological Institute 53: 61.

Bouček, Z., B.R.S. Rao \& S.I. Farooqi (1979). A preliminary review of Pteromalidae (Hymenoptera) of India and adjacent countries. Oriental Insects 12: 433-467.

Brown, B.V. (1993). A further chemical alternative to critical point drying for preparing small (or) large flies, Fly Times 7, p. 10.

Habu, A. (1963). Chalcididae from the Ryuku islands preserved in the Entomological Laboratory of Kyushu University (Hymenoptera). Mushi 37: 115.

Hayat, M. (1981). Taxonomic notes on Indian Encyrtidae (Hymenoptera: Chalcidoidea) - III. Colemania 1: 13-34.

Hayat, M. (1986). Notes on some species of Marietta (Hymenoptera: Aphelinidae), with a key to world species. Colemania 2: 1-18.

Hayat, M. (1998). Aphelinidae of India (Hymenoptera: Chalcidoidea): A taxonomic revision. Memoirs of Entomology, International, Associated Publishers, Gainesville, 13: 416pp.

Hayat, M. (2006). Indian Encyrtidae (Hymenoptera: Chalcidoidea). Department of Zoology, Aligarh Muslim University, Aligarh, India, 496pp.

Hodgson, C.J. (1994). The Scale Insect Family Coccidae: An identification Manual - 6. CAB International, Wallingford, 639pp.

Howard, L.O. (1895). A new genus and species of the Aphelininae. Canadian Entomologist 27(12): 350-351; http://dx.doi. org/10.4039/Ent27351-12

Howard, L.O. (1896). On two interesting new genera of scale insect parasites. Canadian Entomologist 28(6): 165-167; http://dx.doi. org/10.4039/Ent28165-6

Mani, M.S. (1942). Studies on Indian parasitic Hymenoptera II. Indian Journal of Entomology 4: 156.

Mani, M.S. (1989). The Fauna of India and the Adjacent Countries. Zoological Survey of India, Calcutta, 1067pp.

Manickavasagam, S., S. Suresh \& M. Hayat (2001). Anagyrus diversicornis a new record from India (Hymenoptera: Clacidoidea: Encyrtidae). Zoos' Print Journal 16: 494; http://dx.doi.org/10.11609/ JoTT.ZPJ.16.5.494

Motschulsky, V. de (1863). Essai d'un catalogue des insectes de l'lle Ceylon (Suite). Byulleten' Moskovskogo Obshchestva Ispytateley Prirody (Otdel Biologicheskiy) 36: 36-52.

Narendran, T.C. (1986). Family Eucharitidae. In: Rao, B.R.S. \& M. Hayat (eds). The Chalcidoidea (Insecta: Hymenoptera) of India and the Adjacent Countries. Oriental Insects 20: 51-55.

Narendran, T.C. \& T.V. Mini (2000). A review of the genera of Oriental Eunotinae (Hymenoptera: Pteromalidae). Journal of the Zoological Society of Kerala 8\&9: 15-28.

Noyes, J.S. (2000). Encyrtidae of Costa Rica (Hymenoptera: Chalcidoidea), 1. The subfamily Tetracneminae, parasitoids of mealybugs (Homoptera: Pseudococcidae). Memoirs of the American Entomological Institute 62, 355pp.

Noyes, J.S. (2012). Universal Chalcidoidea Database. Available from: <http://www.nhm.ac./Uk/jdsml/research-curation/research/ projects/chalcidoids/synonyms $>$ (Accessed June $12^{\text {th }}, 2012$ ).

Noyes, J.S. \& G.L. Prinsloo (1998). A review of the Afrotropical and Malagasy taxa of Encyrtidae (Hymenoptera: Chalcidoidea) described by J. Risbec (1949-1959). Annales de la Société Entomologique de France, Séries 1-6, 34: 71-97.

Noyes, J.S. \& M. Hayat (1994). Oriental Mealybug Parasitoids of the Anagyrini (Hymenoptera: Encyrtidae). CAB International. The Natural History Museum, London, 554pp.

Riley, C.V., W.H. Ashmead \& L.O. Howard (1894). Report upon the parasitic Hymenoptera of the island of St. Vincent. Journal of the Linnean Society (Zoology) 25: 56-254; http//dx.doi.org/10.1111/ j.1096-3642.1894.tb00982.x

Risbec J. (1959). Encyrtidae de Madagascar (Hym. Chalcid). Bulletin de l'Académie Malgache 35: 27.

Sandlant, G.R. (1978). Slide-mounting fresh specimens of small soft-bodied Homoptera (Mealybugs, scale insects and aphids). The New Zealand Entomologist 6(4): 430-431; http://dx.doi.org/ 10.1080/00779962.1978.9722312

Shafee, S.A. (1972). Indian species of the genus Coccophagus Westwood (Hymenoptera: Aphelinidae). Bulletin of Entomology. Entomological Society of India 13: 25-27.

Sureshan, P.M. (2012). Checklist of Pteromalidae (Insecta: Hymenoptera: Chalcidoidea) of India. Zoological Survey of India, Kozhikode, Kerala, India, 2012, 14pp. Available from: <http://zsi. gov.in/checklist/Checklist\%20Pteromlaidae.pdf> (Accessed 12 June 2012).

Varshney, R.K. (1992). A Checklist of the Scale Insects and Mealybugs of South Asia - Part I. Records of the Zoological Survey of India, Occasional Paper No. 139, 152pp.

Veenakumari, K., P. Mohanraj \& A.K. Bandyopadhyay (1997). Insect herbivores and their natural enemies in the mangals of the Andaman and Nicobar islands. Journal of Natural History 31(7): 1105-1126; http://dx.doi.org/10.1080/00222939700770581

Verma, M., M. Hayat \& S.I. Kazmi (2002). The species of Elasmus from India (Hymenoptera: Chalcidoidea: Eulophidae). Oriental Insects 36(1): 245-306; http://dx.doi.org/10.1080/00305316.2002.10417 334 
Walker, F. (1834). Monographia chalciditum (continued). Entomologica Magazine 2: 35

Walker, F. (1846). List of the specimens of Hymenopterous insects in the collection of the British Museum. Part 1 Chalcidites, London, $85 \mathrm{pp}$.

Williams, D.J. (2004). Mealybugs of Southern Asia. The Natural History Museum and Southdene SDN. BHD. Kaula Lumpur, Malaysia, 896pp.

Xiao, H. \& D.W. Huang (2001). A review of Eunotinae (Hymenoptera: Chalcidoidea: Pteromalidae) from China. Journal of Natural History 35(1): 1587-1605; http://dx.doi.org/ $10.1080 / 002229301317092342$ 